Drug Deliv Transl Res. 2018 February ; 8(1): 226-238. doi:10.1007/s13346-017-0454-8.

\title{
Alginate-liposomal construct for bupivacaine delivery and MSC function regulation
}

\author{
Mollie S. Davis ${ }^{1}$, Ileana Marrero-Berrios ${ }^{1}$, Isabel Perez ${ }^{1}$, Timothy Maguire ${ }^{1}$, Palangat \\ Radhakrishnan ${ }^{2}$, Devasena Manchikalapati ${ }^{2}$, Joseph SchianodiCola ${ }^{2}$, Hattiyangangadi \\ Kamath $^{2}$, Rene S. Schloss ${ }^{1}$, Joel Yarmush ${ }^{2}$ \\ ${ }^{1}$ Department of Biomedical Engineering, Rutgers, The StateUniversity of New Jersey, 599 Taylor \\ Road, Piscataway, NJ 08805, USA \\ ${ }^{2}$ Department of Anesthesiology, New York Methodist Hospital, Brooklyn, NY, USA
}

\begin{abstract}
Mesenchymal stromal cell (MSC) therapies have become potential treatment options for multiple ailments and traumatic injuries. In the clinical setting, MSC are likely to be co-administered with local anesthetics (LA) which have been shown to have dose- and potency-dependent detrimental effects on the viability and function of cells. We previously developed and characterized a sustained-release LA delivery formulation comprised of alginate-encapsulated liposomal bupivacaine. The current studies were designed to evaluate the effect of this formulation on the secretion of three key MSC regulatory molecules, interleukin 6 (IL-6), prostaglandin E2 (PGE2), and transforming growth factor-beta 1 (TGF- $\beta 1$ ). MSCs were treated with several bupivacaine formulations-bolus, liposome, or alginate-liposome construct (engineered construct) -in the presence or absence of inflammatory stimulus to stimulate an injured tissue environment. Our results indicated that compared to bolus or liposomal bupivacaine, the engineered construct preserved or promoted MSC anti-inflammatory PGE2 secretion; however, the engineered construct did not increase TGF- $\beta 1$ secretion. Bupivacaine release profile analyses indicated that mode of drug delivery controlled the LA concentration over time and pathway analysis identified several shared and cytokine-specific molecular mediators for IL-6, PGE2, and TGF- $\beta 1$ which could explain differential MSC secretion responses in the presence of bupivacaine. Collectively, these studies support the potential utility of alginate-encapsulated LA constructs for anti-inflammatory cell therapy co-administration and indicate that mode of local anesthetic delivery can significantly alter MSC secretome function.
\end{abstract}

\section{Keywords}

Controlled release; Local anesthetic; Mesenchymal stromal cell

Rene S. Schloss schloss@ soemail.rutgers.edu.

Compliance with ethical standards The experiments published in this manuscript comply with current laws of the country in which they were performed.

Conflict of interest The authors declare that they have no conflict of interest. 


\section{Introduction}

Local anesthetics (LA) are commonly used to mitigate pain associated with surgical and non-surgical procedures, thereby promoting faster and more efficient patient recovery [1]. These reversible nerve blocks provide pain relief [2,3], which helps patients both during procedures and during post-surgical healing periods. However, despite their advantages, LA have also been shown to have detrimental effects on non-target cells and tissues. For example, multiple studies have demonstrated that common amide LA, such as bupivacaine, have cytotoxic effects in potency-, concentration-, and time-dependent manners on neurons [4] and non-target cells such as chondrocytes [5, 6], fibroblasts [7], and macrophages [8]; which indicates that LA could significantly affect healing and regeneration processes. Therefore, as mesenchymal stromal cell (MSC) therapies become more popular treatment options for a wide range of diseases and traumatic injuries such as autoimmune conditions, wound healing, osteoarthritis, and neurodegenerative disorders and traumas [9], the interest in understanding the effects of co-administered LA has increased $[5,10,11]$.

MSCs are a heterogeneous population of multipotent, adult stem-like cells which can be isolated from multiple tissue sources [12-14]. These cells are non-immunogenic, have migratory potential, and control many physiological functions, which are largely mediated by paracrine secretion [12, 14-17]. LA affect MSC function via secretome and other changes [18], resulting in reduced proliferation [11], differentiation potential [6], lipid metabolism [11], mitochondrial respiration [11], adhesion phenotype [5], and viability $[5,8,10,11,18]$. Recent studies in our laboratory demonstrated that bolus bupivacaine reduced MSC prostaglandin E2 (PGE2) secretion, a key factor responsible for attenuating inflammatory M1 macrophage activity, and also converting macrophages from pro-inflammatory to anti-inflammatory M2 cells [8]. Therefore, new LA delivery strategies are needed that preserve MSC function while still providing sustained pain relief to the patient.

Previous formulations developed to provide controlled LA release include hydrophobicbased polymers [19], poly(lacticco-glycolic acid) microspheres [20], injectable paste and solid polymers [21], and liposomes [22] among others [23]. From these formulations, only Exparel ${ }^{\circledR}$ (liposomal bupivacaine) has been approved for clinical use by the Food and Drug Administration (FDA). Nevertheless, this product only provides consistent analgesia for $24 \mathrm{~h}$ and has considerable constraints such as dose-dumping which could release toxic concentrations of bupivacaine [24].

While encapsulating bupivacaine into liposomes enables a slower drug release [25, 26], to further extend bupivacaine's release profile, we encapsulated the drug-containing liposomes within an alginate hydrogel, which extended drug release up to 4 days [24]. Previously, we showed that in the presence of $1 \mathrm{mM}$ bolus bupivacaine, MSC function, characterized by interleukin 6 (IL-6) and PGE2 secretions, was impaired [27]. Therefore, in order to evaluate the effect of our construct on MSC secretory function, we compared and quantified the MSC secretory profiles of these functionally distinct moieties, IL-6, PGE2, and TGF- $\beta 1$, in co-culture with bolus, liposome, or alginate-liposome constructs. Our results indicate that LA presentation mediates unique temporal secretory responses. These studies support the 
potential utility of alginate-encapsulated LA constructs for cell therapy co-administration but also suggest that the mode of LA administration should be evaluated for functionally specific clinical outcomes.

\section{Materials and methods}

\section{Chemicals and reagents}

Bupivacaine and other chemicals were purchased from Sigma Aldrich (Oakville, Ontario, Canada), unless otherwise stated. All cell culture reagents were purchased from Thermo Fisher Scientific (Waltham, MA), unless otherwise stated.

\section{Liposomes containing bupivacaine}

The liposomes were formed using a dehydration-rehydration protocol, as previously described by Maguire et al. [24]. Briefly, hydrogenated soy phosphatidylcholine (Avanti Polar Lipids, Alabaster, AL) and cholesterol were combined in a 7:3 $\mathrm{M}$ ratio in a round bottom flask to create a lipid and dried via rotovap under vacuum. The lipid film was re-suspended with distilled water and incubated while spinning for $2 \mathrm{~h}$ at $55^{\circ} \mathrm{C}$. The resulting solution was snap frozen and lyophilized overnight. The lipid was then suspended in $70 \mathrm{mg} / \mathrm{mL}$ bupivacaine- $\mathrm{HCl}$ and extruded through polycarbonate membranes 21 times to produce small $200-\mu \mathrm{m}$ unilamellar liposomes. The solution was eluted through a Sephadex G-50 size exclusion column with $0.9 \%$ saline to purify the liposomes.

\section{Alginate encapsulation of liposomes}

Alginate encapsulation of liposomes was done as previously described [24]. Briefly, $2.2 \mathrm{wt} \%$ ultrapure alginic acid (MW 100,000-200,000 g/mol, G content 65-70\%, FMC Biopolymer AS, Sandvika, Norway) was prepared by using ultrapure water and stirring the solution at 65 ${ }^{\circ} \mathrm{C}$. The alginate was filtered using a $0.22-\mu \mathrm{m}$ pore syringe filter (Fisherbrand). Bupivacaineloaded liposomes were mixed, in various volumes based on the target drug concentration of $1 \mathrm{mM}$, with2.2 wt\% alginate and deposited on Transwell inserts (Corning). Then, the inserts containing the liposome alginate mixture were submerged and left to polymerize as a hydrogel sheet for $10 \mathrm{~min}$ at room temperature in a $150 \mathrm{~mL}$ bath containing $100 \mathrm{mM} \mathrm{CaCl}_{2}$, $145 \mathrm{mM} \mathrm{NaCl}$, and $10 \mathrm{mM}$ MOPS. The hydrogels were washed with PBS before they were added to cells.

\section{MSC culture and treatment conditions}

Human bone marrow-derived MSCs (Institute for Regenerative Medicine, Texas A\&M College of Medicine) were cultured as previously described by Maguire, et al. [24]. Briefly, MSCs were thawed at passage 2 and cultured as a monolayer at 300,000 cells in a T-175 flask in a humidified $37^{\circ} \mathrm{C}, 5 \% \mathrm{CO}_{2}$ incubator [18]. Cells were cultured in Minimum Essential medium a containing no deoxy- or ribo-nucleotides, and supplemented with 10\% fetal bovine serum (FBS) (Atlanta Biologicals, Flowery Branch, GA), 2 mM L-glutamine, $1 \mathrm{ng} / \mathrm{mL}$ basic fibroblast growth factor, $100 \mathrm{U} / \mathrm{mL}$ penicillin, and $100 \mathrm{mg} / \mathrm{mL}$ streptomycin. Cells were grown until 70\% confluence, trypsinized, and plated at 12,000 cells/well $\left(6000 \mathrm{cells} / \mathrm{cm}^{2}\right)$ in a 24-well plate. Cells were left to attach overnight before starting the experiments. 
Cell culture media was replaced with fresh fully supplemented media or activation media containing $25 \mathrm{ng} / \mathrm{mL}$ of TNF- $a$ and IFN- $\gamma$ (R\&D Systems, Minneapolis, MN, USA). Then, Transwell inserts (Corning) containing the different treatment conditions (media, 1 $\mathrm{mM}$ bolus bupivacaine, $1 \mathrm{mM}$ bupivacaine-loaded liposomes, or $1 \mathrm{mM}$ alginate-liposome construct) were added to the MSC cultures and incubated for 24,48 , or $96 \mathrm{~h}$ in a humidified $37{ }^{\circ} \mathrm{C}, 5 \% \mathrm{CO}_{2}$ incubator.

\section{Cytokine measurement}

Cell culture supernatants collected from MSC and co-cultures were thawed and tested for different cytokines using enzyme-linked immunosorbent assays. IL-6, TNF- $\alpha$, TGF- $\beta 1$ (Biolegend, San Diego, CA, USA), and PGE2 (Cayman Chemical, Ann Arbor, MI, USA) were all measured according to the manufacturer's instructions. Absorbances were measured using a microplate reader (DTX880 Multimode Detector, Beckman Coulter).

\section{Pathway analysis}

Differentially secreted cytokines were analyzed for overlapping and interacting molecular networks, pathways, and cellular functions using Ingenuity Pathway Analysis software (IPA, QIAGEN, Redwood City, CA, USA). Custom pathways were then established to link anesthetics and deferentially secreted proteins by overlapping upstream regulators using My Pathways and Path Designer functions [27].

\section{Bupivacaine release profile data analysis}

Approximate bupivacaine concentrations at different time points for the three setups were determined using previously published data by Maguire et al. [24]. A computational model was developed using a bupivacaine half-life of $2.7 \mathrm{~h}$ [28]. For bolus, $1 \mathrm{mM}$ was assumed to be the concentration at $t=0$, with no additional bupivacaine released afterwards. Therefore, the decrease in bolus concentration is due solely to degradation. For the sustained release systems-liposomal bupivacaine and the engineered constructdifferences in concentrations were calculated in intervals to mimic the in vitro environment. For liposomal bupivacaine, concentrations were calculated for 8-h increments until $48 \mathrm{~h}$. From 48 to $96 \mathrm{~h}$, it was assumed that no additional bupivacaine was released (based on data by Maguire et al. [24]). For the construct, concentrations were calculated for 8-h increments until $96 \mathrm{~h}$. Bupivacaine concentrations at each increment, following application of the half-life, were added together to give a total bupivacaine concentration at each time point for each system. Correlations were calculated using MATLAB version R2015a (Mathworks, Natick, MA, USA).

\section{Statistics}

Statistical differences among the data were determined using Student's $t$ test for unpaired data or analysis of variance (ANOVA) followed by Fisher's least significant difference (LSD) post hoc analysis with a significance level of $a=0.05$ in Kaleida-Graph software version 4.1 (Synergy Software, Reading, PA, USA). Data points represent the mean \pm the standard error of the mean (SEM) for the indicated number of independent observations $(n)$. 


\section{Results}

\section{Effect of engineered hydrogel-liposome construct on basal MSC secretion}

MSC anti-inflammatory function is largely controlled by their secretome, which is particularly sensitive to micro-environmental changes. Therefore, initial studies were designed to compare and quantify secretion of two molecules, IL-6 and PGE2. IL-6 is constitutively secreted by MSC in large amounts regardless of their state, and is therefore a good indicator of overall MSC function [18], while PGE2 has a prominent role in regulating anti-inflammatory MSC function. As indicated in Fig. 1a, while MSC secretion in the presence of both bolus and liposome bupivacaine were almost identical to control, incubation with the engineered construct resulted in increased IL-6 secretion over time, with a significant increase relative to all other conditions at $96 \mathrm{~h}$. The rate of IL-6 secretion, indicated by the slope of the line between the points, was relatively consistent until 48 $\mathrm{h}$, after which the rate of IL-6 secretion in the construct increased relative to that of the other conditions (Fig. 1b). In general, these studies indicated that while IL-6 concentrations continued to increase throughout the culture period, LA delivery system had a significant effect on IL-6 MSC secretion profiles.

While IL-6 can promote both pro- and anti-inflammatory functions, depending on the tissue state [29, 30], MSC anti-inflammatory function is largely mediated by specific secreted products including the small-molecule PGE2 [17] which is directly responsible for both attenuating pro-inflammatory M1 macrophages and promoting anti-inflammatory alternative M2 macrophages. We previously showed that bolus LA can negatively affect MSC PGE2 secretion [18]. Therefore, in order to test the feasibility of protecting MSC function with our engineered hydrogel-liposome construct, we measured the PGE2 concentration from bupivacaine-treated MSC supernatants. As shown in Fig. 2, after $24 \mathrm{~h}$ of exposure to bolus and liposomal bupivacaine, the PGE2 concentration was significantly lower than media controls. However, while cells exposed to bolus bupivacaine recovered over time, secreting more PGE2 at $96 \mathrm{~h}$, the cells exposed to the liposomal bupivacaine recovered slightly, but not above media controls. In contrast, MSC exposed to the engineered hydrogel-liposome construct continued to secrete PGE2, with significantly higher secretion levels at the 96-h time point.

\section{Effect of engineered hydrogel-liposome construct on activated MSC secretion}

Since the alginate-liposome construct will be used in a peri-operative setting, it is expected to encounter an inflammatory environment. IL- 6 has been shown to be secreted by both nonactivated and activated MSCs, allowing it to be used as an indicator of MSC function even in inflammatory settings [18]. As indicated in Fig. 3a, MSC activated with IFN- $\gamma /$ TNF-a significantly increased IL-6 secretion at 48 and $96 \mathrm{~h}$ compared to all other conditions. The rate of IL-6 secretion was similar for bolus, liposome, and media controls, whereas it was greatly increased in the construct anesthetic condition (Fig. 3b).

Next, we assessed the effect of LA presentation on activated MSC secretion of PGE2. Consistent with that of our previous studies [18], we demonstrated that in the presence of 1 $\mathrm{mM}$ bolus bupivacaine, PGE2 secretion from TNF-a/INF- $\gamma$ activated MSC did not decrease 
after $48 \mathrm{~h}$. However, after both 48 and $96 \mathrm{~h}$ of culture, activated MSC had significantly higher PGE2 secretion over time in the presence of the alginate-liposome construct (Fig. 4).

\section{Effect of engineered hydrogel-liposome construct on MSC TGF- $\beta 1$ secretion}

In addition to their immunomodulatory functions, MSCs also promote tissue repair and regeneration [31]. For example, the secretion of multiple factors, including transforming growth factor-beta (TGF- $\beta 1$ ), facilitates processes such as wound healing [32] and chondrogenesis [33]. Due to the possible site applications for a sustained-release anestheticMSC therapy, and given the effect of LA administration mode on PGE-2 secretion, we analyzed TGF- $\beta 1$ to determine if the construct preserved potential MSC chondrogenic function as well.

As indicated in Fig. 5a, MSC TGF- $\beta 1$ secretion was reduced by the construct relative to bolus bupivacaine administration; however, this was only significant at $48 \mathrm{~h}$. At $24 \mathrm{~h}$, both bolus and construct demonstrated increased TGF- $\beta 1$ secretion compared to media control, and by $96 \mathrm{~h}$, relative TGF- $\beta 1$ values were similar, regardless of the anesthetic vehicle. The rate of TGF- $\beta 1$ secretion, as indicated by the slope of the line in Fig. $5 \mathrm{~b}$, was relatively consistent for bolus, lipo-some, and construct between 48 and $96 \mathrm{~h}$. The media control demonstrated an increased rate of secretion at 48 and $96 \mathrm{~h}$, indicating that LA, regardless of presentation mode, reduced TGF- $\beta 1$ secretion rate at later time points.

In addition, we examined TGF- $\beta 1$ secretory responses in an inflammatory milieu. As indicated in Fig. 6, activated MSC TGF- $\beta 1$ secretion was significantly reduced in the construct anesthetic condition compared to the bolus dose condition at $96 \mathrm{~h}$. No other time points or conditions were significantly different.

\section{Pathway analysis}

To investigate the link between secretion changes in an inflammatory milieu and bupivacaine exposure, we performed a pathway analysis using the Ingenuity Pathway Analysis (IPA) software. By using IPA, previous studies performed by our group suggested that bupivacaine affects MSC cellular functions and secretion patterns depending on their stimulation state [27]. To further understand the MSC secretion patterns observed here, we searched for overlapping and interacting molecular signatures using IPA and its functions My Pathways and Path Designer. Using these functions, which establish relationships between gene products/ molecules by the action of mediators using the Ingenuity Knowledge Base, we were able to identify molecular mediators that are affected by bupivacaine and which, in turn, may differentially affect IL-6, PGE2, and TGF- $\beta 1$ behavior. As specified by IPA, IL-6, PGE2, and TGF- $\beta 1$ share several molecular mediators, including p38 mitogen-activated protein kinases (p38 MAPK), reactive oxygen species (ROS), and calcium $\left(\mathrm{Ca}^{2+}\right)$ that under the effects of bupivacaine can exert changes in their expression and secretion. In addition, TGF- $\beta 1$ and IL6 shared jun-proto-oncogene/activator protein 1 (JUN) while TGF - $\beta 1$ and PGE2 share dhydroxyprostaglandin dehydrogenase 15-(NAD) (HPGD). IL-6 had two unique molecular signatures junD protooncogene (JUND) and FOS like 1/AP-1 transcription factor subunit (FOSL-1). In addition, PGE2 is a molecular mediator of TGF- $\beta 1$ and IL-6. With this information, we were able to build a custom pathway showing these 
molecular interactions (Fig. 7). While the three secreted molecules share common molecular mediators, they also exhibit unique regulatory elements, suggesting differential bupivacaine regulatory effects.

\section{Bupivacaine release profiles}

Given the fact that bupivacaine presentation mode had a significant effect on MSC secretions over time, we estimated the release profiles based on the measured released LA concentrations [24] and the half-life of bupivacaine [28] in order to gain further insight into the factors that may control differential bupivacaine effects on MSC secretion. Bupivacaine concentrations over time were calculated, as seen in Fig. 8. Bolus concentrations drop drastically, with the concentration at $8 \mathrm{~h}$ less than $13 \%$ of the original dose. By $24 \mathrm{~h}, 0.2 \%$ of the original dose remains in solution. Liposomal concentration peaks at $8 \mathrm{~h}$, with $\sim 33 \%$ of the total dose released. Within $24 \mathrm{~h}, 69 \%$ of the total intended concentration is released [24], and the amount in solution remains at $\sim 26 \%$. By $48 \mathrm{~h}$, bupivacaine concentration drops to $2 \%$. In contrast, construct concentration remains fairly constant for most of the 96 $\mathrm{h}$ analyzed. For the first $24 \mathrm{~h}$, the concentrations peaks at $10 \%$ and then remains around $8 \%$. The concentration drops to $6 \%$ at $48 \mathrm{~h}$ and to $\sim 2 \%$ at $96 \mathrm{~h}$.

In order to further understand the effect of different anesthetic conditions on MSC secretion, data correlations were established using the calculated concentrations (Fig. 8) and the secretion profiles for IL-6, PGE2, and TGF- $\beta 1$. The correlations between secretion concentration and either bupivacaine or time are described in Figs. 9 and 10, respectively. Values are listed in Tables 1 and 2. In general, IL-6 levels trended higher over time, and lower in the presence of increased bupivacaine concentration, regardless of the presentation mode. PGE2 concentrations trended lower over time in all cases except in the presence of the engineered construct. TGF- $\beta 1$ concentrations trended higher over time, and lower in the presence of increased bupivacaine in all cases. This analysis supports the conclusion that bupivacaine, as well as its presentation mode and release profile, has differential effects on individual MSC secretome components.

\section{Discussion}

Due to their regenerative and immunomodulatory potential, MSCs have been evaluated in the past several years as potential cell-based therapeutics for many applications including joint repair procedures $[5,6,34,35]$. These procedures often necessitate administration of LA to minimize patient pain and discomfort. Therefore, understanding the effects of LA on MSC function has become critical to guarantee optimal clinical outcomes [5]. Several studies, including our own, have demonstrated that relevant clinical doses of LA have adverse effects on MSC therapeutic potential. As a result, the development of new LA formulations that allow for co-administration of MSC therapeutics and LA is needed. A recent publication by our group described the development and initial characterization of an engineered hydrogel-liposome construct containing alginate-encapsulated liposomal bupivacaine that allows for the sustained release of low doses of bupivacaine for a minimum of 4 days [24]. In addition, after 4 days of continuous culture, the constructs yielded significantly greater MSC viability than either the liposomal bupivacaine or the bolus dose 
of bupivacaine [24]. Preliminary studies indicate the efficacy of construct injection into the site of injury (data not shown). However, the effect of this construct on MSC function was not addressed in those studies.

Therefore, the current studies aimed to investigate the effects of the engineered hydrogelliposome construct on MSC secretory function compared to other bupivacaine formulations currently used in the clinical setting (bolus dose and liposomal bupivacaine). TNF-a/INF$\gamma$-activated and non-activated MSCs were treated with $1 \mathrm{mM}$ of the bolus, liposomal bupivacaine, and construct as previously described [24] for 24,48 , and $96 \mathrm{~h}$ and their supernatants were analyzed.

In order to understand MSC function in the presence of engineered hydrogel-liposome constructs, constitutive IL-6 and immunomodulatory PGE2 levels were analyzed. Previous data showed that a bolus dose of $1 \mathrm{mM}$ bupivacaine significantly reduced IL-6 secretion compared to media control [18]. Our studies showed that with the engineered hydrogelliposome construct, IL-6 secretion was significantly increased compared to bolus dosing at $96 \mathrm{~h}$. Moreover, IL-6 secretion was also higher in the presence of the construct than for media controls, suggesting that the construct promoted increased activity. To understand why IL-6 was almost twofold higher than media control conditions, we analyzed the effect of alginate on IL-6 secretion and found no effect (data not shown). This suggests that MSC may be stressed by the continual presence of low, but relatively constant, amounts of bupivacaine. Given that low stress levels are known to improve function [36], future studies will examine the expression of stress proteins in the presence of different anesthetic formulations.

Similarly, we have shown that PGE2 secretion is significantly reduced in MSC treated with $1 \mathrm{mM}$ bolus bupivacaine [18]. Since PGE2 is an important modulator of immune cells capable of inducing a phenotypic switch from pro-inflammatory TNF- $a$-secreting M1 macrophages to inflammation-resolving IL-10-secreting M2 macrophages, low PGE2 secretion could functionally impair MSC therapeutic potential. However, MSC exposed to the engineered hydrogel-liposome construct secreted higher levels of PGE2 at all times compared to other LA formulations and media controls. This indicates that the construct not only preserves MSC PGE2 secretory function but also promotes it over time.

Since MSCs have a wide range of functions that are mediated by their secretome, including tissue repair and regeneration, we investigated the effect of the engineered hydrogelliposome construct on MSC's TGF- $\beta 1$ secretion which is a multifunctional growth factor involved in various biological cascades, such as cellular proliferation, differentiation, and migration [37]. MSCs that produce TGF- $\beta 1$ among other regenerative factors could be used as a treatment for degenerative diseases such as osteoarthritis. Interestingly, after $96 \mathrm{~h}$ of continuous culture, the MSC's TGF- $\beta 1$ secretion was impaired for the liposome and engineered hydrogel-liposome construct treatments. In contrast, MSCs exposed to $1 \mathrm{mM}$ bolus bupivacaine secreted TGF- $\beta 1$ levels comparable to that of basal media controls, a secretion pattern that was not observed for IL-6 or PGE2. 
In order to simulate an injured tissue inflammatory environment, MSCs were stimulated with TNF-a/INF- $\gamma$, which allows for analysis of the construct in a more realistic environment. Our studies indicate that the construct elevated IL-6 secretion levels compared to all conditions at 48 and $96 \mathrm{~h}$. Relative IL-6 levels peaked at $48 \mathrm{~h}$ for all conditions. This may be due to an elevated, stressed state for MSC at that time point. While the IL-6 concentration at $96 \mathrm{~h}$ was elevated compared to $48 \mathrm{~h}$ for all conditions (data not shown), the relative concentration compared to media dropped. The drop at $96 \mathrm{~h}$ for all LA conditions may similarly be due to stress response activation, but in this case, the combination of TNF$a / \mathrm{INF}-\gamma$ activation along with LA-mediated activation may have slowed down secretion of IL-6 compared to media controls.

As previously observed for TNF-a/INF- $\gamma$ activated MSC, PGE2 secretion patterns were more variable and did not necessarily correlate with trends observed for non-activated MSC [18]. Overall PGE2 secretion was higher than media controls for all bupivacaine formulations with peak secretion levels after $48 \mathrm{~h}$ of continuous culture. Interestingly, a drastic decrease in PGE2 levels was observed at $96 \mathrm{~h}$ in all conditions. Two possible explanations for this phenomenon may be (1) uptake and eventual degradation of TNF-a/ INF- $\gamma$ over time, as these cytokines have relatively short half-lives (minutes), or(2) MSC exhaustion due to continuous stimulation with activating factors. Further studies where MSCs receive fresh activation factors every day or where MSCs are pre-activated with TNF- $a / I N F-\gamma$ over longer time points are needed to more completely understand these observations.

Correspondingly, MSC activated with TNF- $\alpha$ /NNF- $\gamma$ showed a differential TGF- $\beta 1$ secretion pattern which did not resemble the non-activated MSC pattern. Previous studies have shown that TNF- $\alpha$ and INF- $\gamma$ induce the expression TGF- $\beta 1$ in other cell types [37, 38]. In this study, activated MSC TGF- $\beta 1$ secretion levels at $24 \mathrm{~h}$ were higher than that of the non-activated MSC for all treatment groups. However, after $96 \mathrm{~h}$ of continuous culture in an inflammatory environment, the $1 \mathrm{mM}$ bolus bupivacaine condition had higher TGF- $\beta 1$ secretion than all conditions, followed by basal media controls, liposome treatment, and a significant drop of TGF- $\beta 1$ levels for the construct. We hypothesize that the continuous presence of small doses of bupivacaine might be deleterious for MSC regenerative function and the presence of inflammation might enhance these effects.

To evaluate the relationship between secretome changes in the presence of bupivacaine and inflammation, we performed a pathway analysis using IPA. The molecular signatures evaluated in our studies for overall cell function (IL-6), anti-inflammatory function (PGE2), and regenerative function (TGF- $\beta 1$ ) shared molecular mediators such as p38-MAPK, ROS, and $\mathrm{Ca}^{2+}$ that in the presence of bupivacaine should increase their expression and secretion [39-42]. Some were regulators of each other, such as PGE2 increasing the expression of TGF- $\beta 1[43,44]$ and IL-6 $[45,46]$ in addition to TGF- $\beta 1$ and IL-6 increasing the production of PGE2 [47, 48]. However, the secretion patterns observed in our studies differed for each cytokine and bupivacaine formulation over time. This could be due to differential effects of bupivacaine on the individual molecular mediators found for each cytokine or due to other molecular mediators that have not been identified yet as targets of bupivacaine action. 
Therefore, further studies with MSC are required to elucidate the mechanisms underlying the interaction of LA on cellular function and secretion.

With the purpose of further understanding the MSC responses to the LA formulations, we performed data correlations of cytokine concentrations with LA concentration or time. The correlation data hints to the severity that initial drug concentrations have on MSC function. Bolus dose, which has a high concentration initially, but is almost 0 at $24 \mathrm{~h}$, continued to impede MSC secretion. The hydrogel-liposome construct on the other hand, which maintains a drug concentration of around $8 \%$ for most of the $96 \mathrm{~h}$, maintained MSC secretion throughout the culture period. The IL- 6 correlations, which have higher Pearson $r$ values for the constructs compared to the other conditions, suggests that the initial LA insult affects MSC function even after the anesthetic is degraded and that maintaining a low anesthetic concentration, even for a longer period of time, is preferable to a higher dose for a shorter time frame.

Co-delivery of local anesthetics and MSC alters the MSC secretome. Depending on the method of delivery, some cytokines may be upregulated while others may be downregulated or unchanged. Collectively, our studies indicate that the engineered bupivacaine construct did not impede, and may even promote, MSC anti-inflammatory function, supporting its co-administration with MSC and perhaps other cell therapies. However, the presence of a controlled-release LA formulation may have detrimental effects on MSC regenerative function. Therefore, caution must be exerted when selecting LA formulations to accompany cell therapies depending on the intended cell therapeutic function.

\section{Acknowledgments}

Funding information Support for this work came from grants from the United States Department of Education (Graduate Assistance in Areas of National Need Award P200A150131) and the National Institutes of Health (Ruth L. Kirschstein National Research Service Award T32 GM008339 from the National Institute of General Medical Sciences).

\section{References}

1. Lirk P, Picardi S, Hollmann MW. Local anaesthetics. Eur J Anaesthesiol. 2014;31(11):575-85. 10.1097/eja.0000000000000137. [PubMed: 25192265]

2. Ballieul RJJT, Herregods S, Van Sint Jan P, Wyler B, Vereecke H, Almqvist F, et al. The perioperative use of intra-articular local anesthetics: a review. Acta Anaesthesiol Belg. 2009;6(2):7.

3. Becker DE, Reed KL. Local anesthetics: review of pharmacological considerations. Anesth Prog. 2012;59(2):90-101. 10.2344/0003-3006-59.2.90. [PubMed: 22822998]

4. Perez-Castro R, Patel S, Garavito-Aguilar ZV, Rosenberg A, Recio-Pinto E, Zhang J, et al. Cytotoxicity of local anesthetics in human neuronal cells. Anesth Analg. 2009;108(3):997-1007. 10.1213/ane.0b013e31819385e1. [PubMed: 19224816]

5. Dregalla RC, Lyons NF, Reischling PD, Centeno CJ. Amide-type local anesthetics and human mesenchymal stem cells: clinical implications for stem cell therapy. Stem Cells Transl Med. 2014;3(3): 365-74. 10.5966/sctm.2013-0058. [PubMed: 24436443]

6. Breu A, Eckl S, Zink W, Kujat R, Angele P. Cytotoxicity of local anesthetics on human mesenchymal stem cells in vitro. Arthroscopy. 2013;29(10):1676-84. 10.1016/j.arthro.2013.06.018. [PubMed: 23993145] 
7. Fedder C, Beck-Schimmer B, Aguirre J, Hasler M, Roth-Z'graggen B, Urner M, et al. In vitro exposure of human fibroblasts to local anaesthetics impairs cell growth. Clin Exp Immunol. 2010;162(2): 280-8. 10.1111/j.1365-2249.2010.04252.x. [PubMed: 20819090]

8. Gray A, Marrero-Berrios I, Weinberg J, Manchikalapati D, SchianodiCola J, Schloss RS, et al. The effect of local anesthetic on pro-inflammatory macrophage modulation by mesenchymal stromal cells. Int Immunopharmacol. 2016;33:48-54. 10.1016/j.intimp.2016.01.019. [PubMed: 26854576]

9. Trounson A, McDonald C. Stem cell therapies in clinical trials: progress and challenges. Cell Stem Cell. 2015;17(1):11-22. 10.1016/j.stem.2015.06.007. [PubMed: 26140604]

10. Rahnama R, Wang M, Dang AC, Kim HT, Kuo AC. Cytotoxicity of local anesthetics on human mesenchymal stem cells. J Bone Joint Surg Am. 2013;95(2):132-7. 10.2106/JBJS.K.01291. [PubMed: 23324960]

11. Lucchinetti PDE, Awad MD, Ahmed E, MDM R, MDPDJ F, PDPH L, et al. Antiproliferative effects of local anesthetics on mesenchymal stem cells potential implications for tumor spreading and wound healing. Anesthesiology. 2012;116(4):841-56. 10.1097/ALN.0b013e31824babfe. [PubMed: 22343474]

12. Barminko J, Kim JH, Otsuka S, Gray A, Schloss R, Grumet M, et al. Encapsulated mesenchymal stromal cells for in vivo transplantation. Biotechnol Bioeng. 2011;108(11):2747-58. 10.1002/ bit.23233. [PubMed: 21656712]

13. Pittenger MF, Mackay AM, Beck SC, Jaiswal RK, Douglas R, Mosca JD, et al. Multilineage potential of adult human mesenchymal stem cells. Science. 1999;284(5411):143-7. 10.1126/ science.284.5411.143. [PubMed: 10102814]

14. Meirelles Lda S, Fontes AM, Covas DT, Caplan AI. Mechanisms involved in the therapeutic properties of mesenchymal stem cells. Cytokine Growth Factor Rev. 2009;20(5-6):419-27. 10.1016/j.cytogfr.2009.10.002. [PubMed: 19926330]

15. Gray A, Maguire T, Schloss R, Yarmush ML. Identification of IL-1 $\beta$ and LPS as optimal activators of monolayer and alginate-encapsulated mesenchymal stromal cell immunomodulation using design of experiments and statistical methods. Biotechnol Prog. 2015;31(4):1058-70. 10.1002/ btpr.2103. [PubMed: 25958832]

16. Di Nicola M, Carlo-Stella C, Magni M, Milanesi M, Longoni PD, Matteucci P, et al. Human bone marrow stromal cells suppress T-lymphocyte proliferation induced by cellular or nonspecific mitogenic stimuli. Blood. 2002;99(10):3838-43. 10.1182/blood.V99.10.3838. [PubMed: 11986244]

17. Barminko JA, Nativ NI, Schloss R, Yarmush ML. Fractional factorial design to investigate stromal cell regulation of macrophage plasticity. Biotechnol Bioeng. 2014;111(11):2239-51. 10.1002/ bit.25282. [PubMed: 24891120]

18. Gray A, Marrero-Berrios I, Ghodbane M, Maguire T, Weinberg J, Manchikalapati D, et al. Effect of local anesthetics on human mesenchymal stromal cell secretion. Nano Life. 2015;5(02):1550001-14. 10.1142/S1793984415500014. [PubMed: 26539251]

19. Jain R, Shah NH, Malick AW, Rhodes CT. Controlled drug delivery by biodegradable poly(ester) devices: different preparative approaches. Drug Dev Ind Pharm. 1998;24(8):703-27. 10.3109/03639049809082719. [PubMed: 9876519]

20. Blanco MD, Bernardo MV, Gomez C, Muniz E, Teijon JM. Bupivacaine-loaded comatrix formed by albumin microspheres included in a poly(lactide-co-glycolide) film: in vivo biocompatibility and drug release studies. Biomaterials. 1999;20(20):1919-24. 10.1016/S0142-9612(99)00092-7. [PubMed: 10514068]

21. Shikanov A, Domb AJ, Weiniger CF. Long acting local anesthetic-polymer formulation to prolong the effect of analgesia. J Control Release. 2007;117(1):97-103. 10.1016/j.jconrel.2006.10.014. [PubMed: 17137669]

22. Cohen SM. Extended pain relief trial utilizing infiltration of Exparel®, a long-acting multivesicular liposome formulation of bupivacaine: a phase IV health economic trial in adult patients undergoing open colectomy. J Pain Res. 2012;5:567-72. 10.2147/JPR.S38621. [PubMed: 23204866]

23. Weiniger CF, Golovanevski L, Domb AJ, Ickowicz D. Extended release formulations for local anaesthetic agents. Anaesthesia. 2012;67(8):906-16. 10.1111/j.1365-2044.2012.07168.x. [PubMed: 22607613] 
24. Maguire T, Davis M, Marrero-Berrios I, Zhu C, Gaughan C, Weinberg J, Manchikalapati D, SchianodiCola J, Kamath H, Schloss R, Yarmush J. Control release anesthetics to enable an integrated anesthetic-mesenchymal stromal cell therapeutic. Int J Anesth Pain Med. 2016. 10.21767/2471-982X.100012.

25. Lambrechts M, O’Brien MJ, Savoie FH, You Z. Liposomal extended-release bupivacaine for postsurgical analgesia. Patient Preference and Adherence. 2013;7:885-90. 10.2147/PPA.S32175. [PubMed: 24043932]

26. Rogobete AF, Bedreag OH, Sărăndan M, Păpurică M, Preda G, Dumbuleu MC, et al. Liposomal bupivacaine-new trends in anesthesia and intensive care units. Egypt J Anaesthesia. 2015;31(1): 89-95. 10.1016/j.egja.2014.12.004.

27. Gray A, Marrero-Berrios I, Ghodbane M, Maguire T, Weinberg J, Manchikalapati D, et al. Effect of local anesthetics on human mesenchymal stromal cell secretion. Nano Life. 2015;05(02):1550001-14. 10.1142/s1793984415500014.

28. FDA (2012) Bupivacaine hydrochloride injection, USP. http://www.accessdata.fda.gov/ drugsatfda_docs/label/2012/018692s015lbl.pdf.

29. Xing Z, Gauldie J, Cox G, Baumann H, Jordana M, Lei XF, et al. IL-6 is an antiinflammatory cytokine required for controlling local or systemic acute inflammatory responses. J Clin Investig. 1998;101(2):311-20. 10.1172/JCI1368. [PubMed: 9435302]

30. Scheller J, Chalaris A, Schmidt-Arras D, Rose-John S. The pro- and anti-inflammatory properties of the cytokine interleukin-6. Biochimica et Biophysica Acta (BBA) - Mol Cell Res. 2011;1813(5):878-88. 10.1016/j.bbamcr.2011.01.034.

31. Meretoja VV, Dahlin RL, Kasper FK, Mikos AG. Enhanced chondrogenesis in co-cultures with articular chondrocytes and mesenchymal stem cells. Biomaterials. 2012;33(27):6362-9. 10.1016/ j.biomaterials.2012.05.042. [PubMed: 22695067]

32. Faulknor RA, Olekson MA, Nativ NI, Ghodbane M, Gray AJ, Berthiaume F. Mesenchymal stromal cells reverse hypoxia-mediated suppression of a-smooth muscle actin expression in human dermal fibroblasts. Biochem Biophys Res Commun. 2015;458(1):8-13. 10.1016/j.bbrc.2015.01.013. [PubMed: 25625213]

33. Hunziker EB, Driesang IMK, Morris EA. Chondrogenesis in cartilage repair is induced by members of the transforming growth factor-beta superfamily. Clin Orthop Relat Res. 2001;391:S171-S81. 10.1097/00003086-200110001-00017.

34. Davatchi F, Abdollahi BS, Mohyeddin M, Shahram F, Nikbin B. Mesenchymal stem cell therapy for knee osteoarthritis. Preliminary report of four patients. Int J Rheum Dis. 2011;14(2):211-5. 10.1111/j.1756-185X.2011.01599.x. [PubMed: 21518322]

35. Abrao J, Fernandes CR, White PF, Shimano AC, Okubo R, Lima GB, et al. Effect of local anaesthetic infiltration with bupivacaine and ropivacaine on wound healing: a placebo-controlled study. Int Wound J. 2012;11(4):379-85. 10.1111/j.1742-481X.2012.01101.x. [PubMed: 23095130]

36. Diaz MF, Vaidya AB, Evans SM, Lee HJ, Aertker BM, Alexander AJ, et al. Biomechanical forces promote immune regulatory function of bone marrow mesenchymal stromal cells. Stem Cells. 2017;9(10)

37. Sullivan DE, Ferris M, Nguyen H, Abboud E, Brody AR. TNF-alpha induces TGF-beta1 expression in lung fibroblasts at the transcriptional level via AP-1 activation. J Cell Mol Med. 2009;13(8B): 1866-76. 10.1111/j.1582-4934.2008.00647.x. [PubMed: 20141610]

38. Nagineni CN, Cherukuri KS, Kutty V, Detrick B, Hooks JJ. Interferon-gamma differentially regulates TGF-beta1 and TGF- beta2 expression in human retinal pigment epithelial cells through JAK-STAT pathway. J Cell Physiol. 2007;210(1):192-200. 10.1002/jcp.20839. [PubMed: 17013806]

39. Chang YC, Hsu YC, Liu CL, Huang SY, MC H, Cheng SP. Local anesthetics induce apoptosis in human thyroid cancer cells through the mitogen-activated protein kinase pathway. PLoS One. 2014;9(2):e89563. 10.1371/journal.pone.0089563. [PubMed: 24586874]

40. Harato M, Huang L, Kondo F, Tsunekawa K, Feng GG, Fan JH, et al. Bupivacaine-induced apoptosis independently of WDR35 expression in mouse neuroblastoma Neuro2a cells. BMC Neurosci. 2012;13(1):149. 10.1186/1471-2202-13-149. [PubMed: 23227925] 
41. Galbes O, Bourret A, Nouette-Gaulain K, Pillard F, Matecki S, Py G, et al. N-acetylcysteine protects against bupivacaine-induced myotoxicity caused by oxidative and sarcoplasmic reticulum stress in human skeletal myotubes. Anesthesiology. 2010;113(3):560-9. 10.1097/ ALN.0b013e3181e4f4ec. [PubMed: 20683254]

42. Irwin W, Fontaine E, Agnolucci L, Penzo D, Betto R, Bortolotto S, et al. Bupivacaine myotoxicity is mediated by mitochondria. J Biol Chem. 2002;277(14):12221-7. 10.1074/jbc.M108938200. [PubMed: 11790774]

43. Capper EA, Roshak AK, Bolognese BJ, Podolin PL, Smith T, Dewitt DL, et al. Effect of local anesthetics by tranilast, SB 252218, a compound demonstrating efficacy in restenosis. J Pharmacol Exp Ther. 2000;295(3):1061-9. [PubMed: 11082441]

44. Mao Y, Sarhan D, Steven A, Seliger B, Kiessling R, Lundqvist A. Inhibition of tumor-derived prostaglandin-e2 blocks the induction of myeloid-derived suppressor cells and recovers natural killer cell activity. Clin Cancer Res. 2014;20(15):4096-106. 10.1158/1078-0432.CCR-14-0635. [PubMed: 24907113]

45. Inflammation Medzhitov R. 2010: new adventures of an old flame. Cell. 2010;140(6):771-6. 10.1016/j.cell.2010.03.006. [PubMed: 20303867]

46. Benito MJ, Veale DJ, FitzGerald O, van den Berg WB, Bresnihan B. Synovial tissue inflammation in early and late osteoarthritis. Ann Rheum Dis. 2005;64(9):1263-7. 10.1136/ard.2004.025270. [PubMed: 15731292]

47. Pilbeam C, Rao Y, Voznesensky O, Kawaguchi H, Alander C, Raisz L, et al. Transforming growth factor-beta1 regulation of prostaglandin $\mathrm{G} / \mathrm{H}$ synthase-2 expression in osteoblastic MC3T3-E1 cells. Endocrinology. 1997;138(11):4672-82. 10.1210/endo.138.11.5495. [PubMed: 9348193]

48. Wakabayashi A, Sawada K, Nakayama M, Toda A, Kimoto A, Mabuchi S, et al. Targeting interleukin-6 receptor inhibits preterm delivery induced by inflammation. Mol Hum Reprod. 2013;19(11): 718-26. 10.1093/molehr/gat057. [PubMed: 23969038]

Drug Deliv Transl Res. Author manuscript; available in PMC 2018 November 06. 

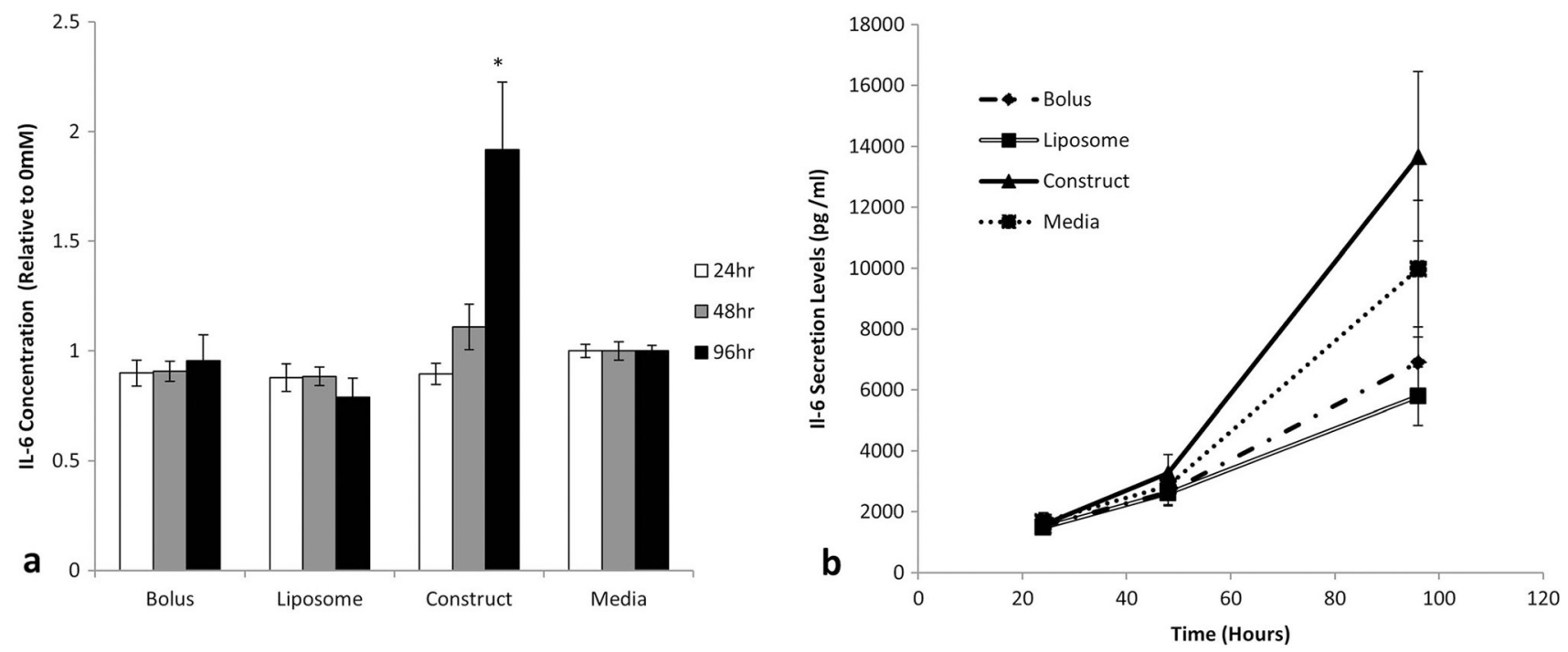

Fig. 1.

Alginate-liposome construct preserves MSC IL-6 secretion. Levels of IL-6 quantified in cell culture supernatants collected from MSCs treated for 24,48 , and $96 \mathrm{~h}$ with $1 \mathrm{mM}$ bolus dose of bupivacaine liposomal bupivacaine or alginate-liposome construct. a IL-6 concentrations were normalized to corresponding basal medium controls $(0 \mathrm{mM})$. b IL-6 secretion over time. Each bar (a) or point (b) is the mean \pm SEM of $n=14$ independent observations. An asterisk indicates $<0.001$ all conditions at time point 

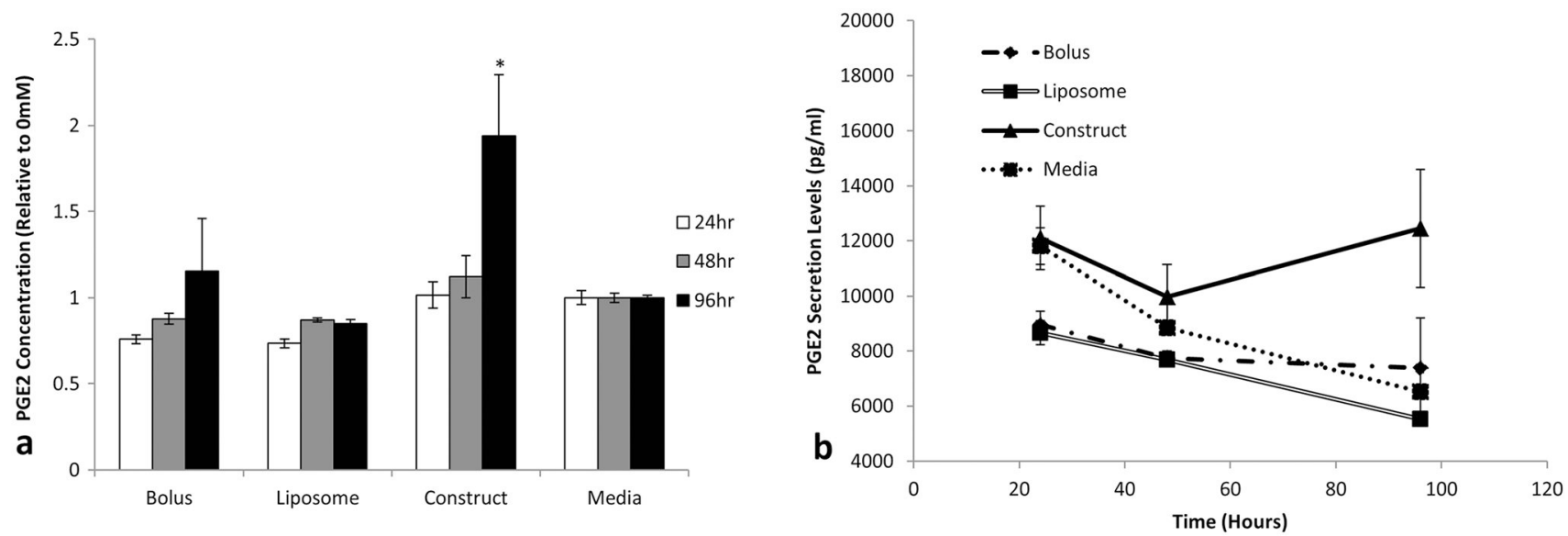

Fig. 2.

Alginate-liposome construct preserves MSC PGE2 secretion. Levels of PGE2 quantified in cell culture supernatants collected from MSCs treated for 24, 48, and $96 \mathrm{~h}$ with 1 $\mathrm{mM}$ bolus dose of bupivacaine, liposomal bupivacaine, or alginate-liposome construct. a PGE2 concentrations were normalized to corresponding basal medium controls $(0 \mathrm{mM})$. b Baseline PGE2 levels. Each bar (a) or point (b) is the mean \pm SEM of $n=9$ independent observations. An asterisk indicates $<0.005$ all conditions at time point 

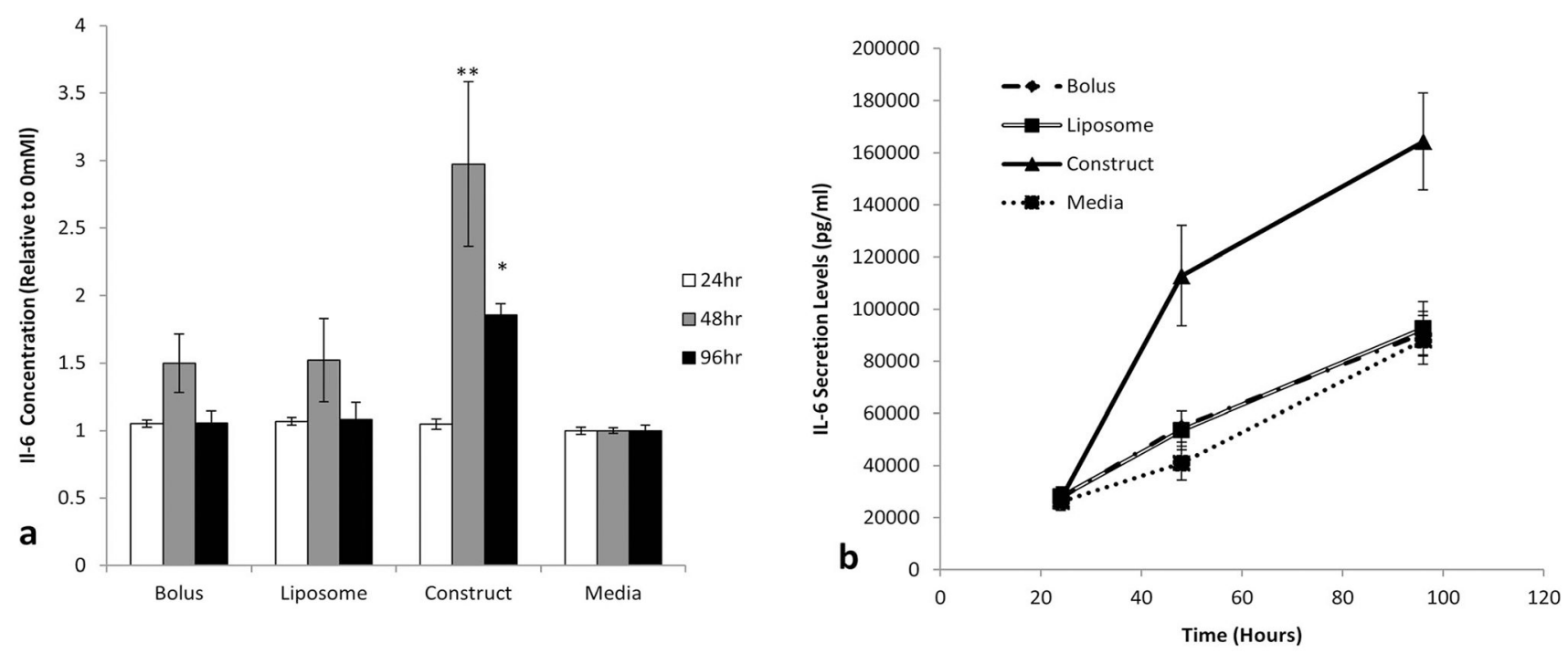

Fig. 3.

Alginate-liposome construct preserves activated MSC IL-6 secretion. Levels of IL-6 quantified in cell culture supernatants collected from MSCs treated for 24, 48, and $96 \mathrm{~h}$ with $1 \mathrm{mM}$ bolus dose of bupivacaine, liposomal bupivacaine, or alginate-liposome construct with TNF-a/INF- $\gamma(25 \mathrm{ng} / \mathrm{mL})$. a IL-6 concentrations were normalized to corresponding basal medium controls $(0 \mathrm{mM})$.). b Baseline IL-6 levels. Each bar (a) or point (b) is the mean \pm SEM of $n=9$ independent observations. An asterisk indicates $<0.05$ all conditions at time point. Two asterisks indicate $<0.001$ all conditions at time point 

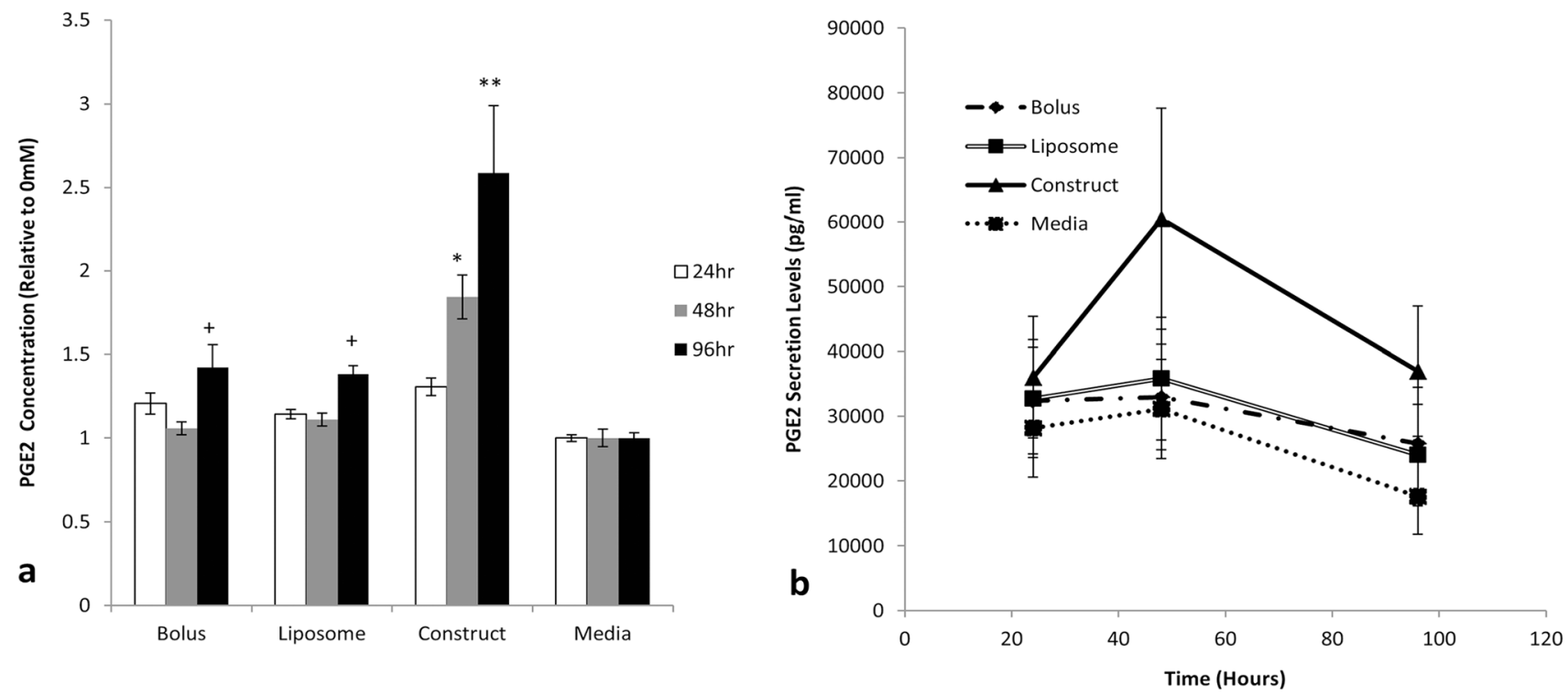

Fig. 4.

Alginate-liposome construct preserves activated MSC PGE2 secretion. Levels of PGE2 quantified in cell culture supernatants collected from MSCs treated for 24, 48, and $96 \mathrm{~h}$ with $1 \mathrm{mM}$ bolus dose of bupivacaine, liposomal bupivacaine, or alginate-liposome construct with TNF-a/INF- $\gamma(25 \mathrm{ng} / \mathrm{mL})$. a PGE2 concentrations were normalized to corresponding basal medium controls $(0 \mathrm{mM})$.). b Baseline PGE2 levels. Each bar (a) or point (b) is the mean \pm SEM of $n=6$ independent observations. An asterisk indicates $<0.0005$ all conditions at time point. Two asterisks indicate $<0.001$ all conditions at time point. A plus sign indicates $<0.05$ compared to media at time point 

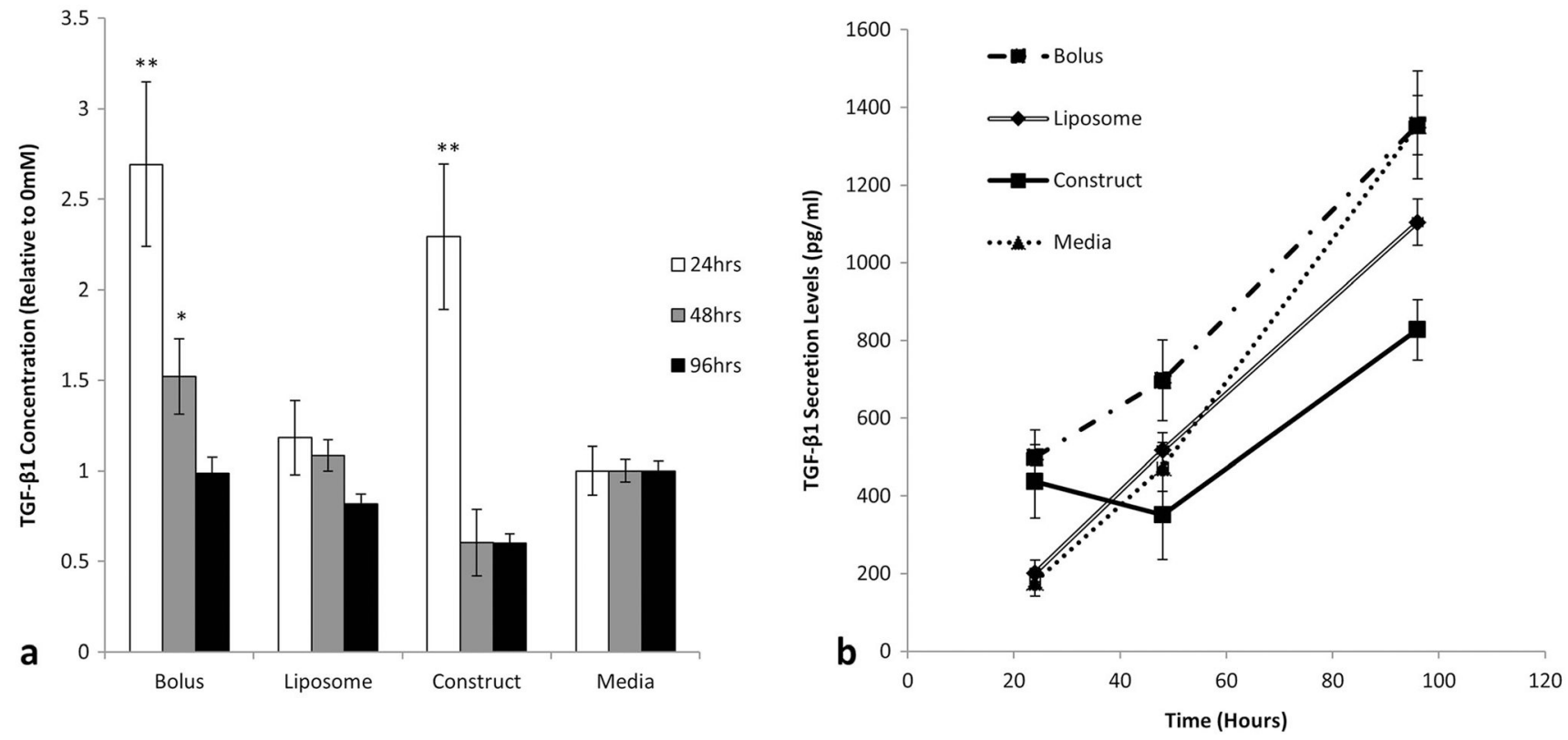

Fig. 5.

Alginate-liposome construct decreases MSC TGF- $\beta 1$ secretion. Levels of TGF- $\beta 1$ quantified in cell culture supernatants collected from MSCs treated for 24, 48, and $96 \mathrm{~h}$ with $1 \mathrm{mM}$ bolus dose of bupivacaine, liposomal bupivacaine, or alginate-liposome construct. a TGF- $\beta 1$ concentrations were normalized to corresponding basal medium controls $(0 \mathrm{mM})$. b TGF- $\beta 1$ secretion over time. Each bar (a) or point (b) is the mean \pm SEM of $n=6-9$ independent observations. Some observations were not detected. An asterisk indicates $<0.05$ construct and media at time point. Two asterisks indicate $<0.0002$ media and liposome at time point 

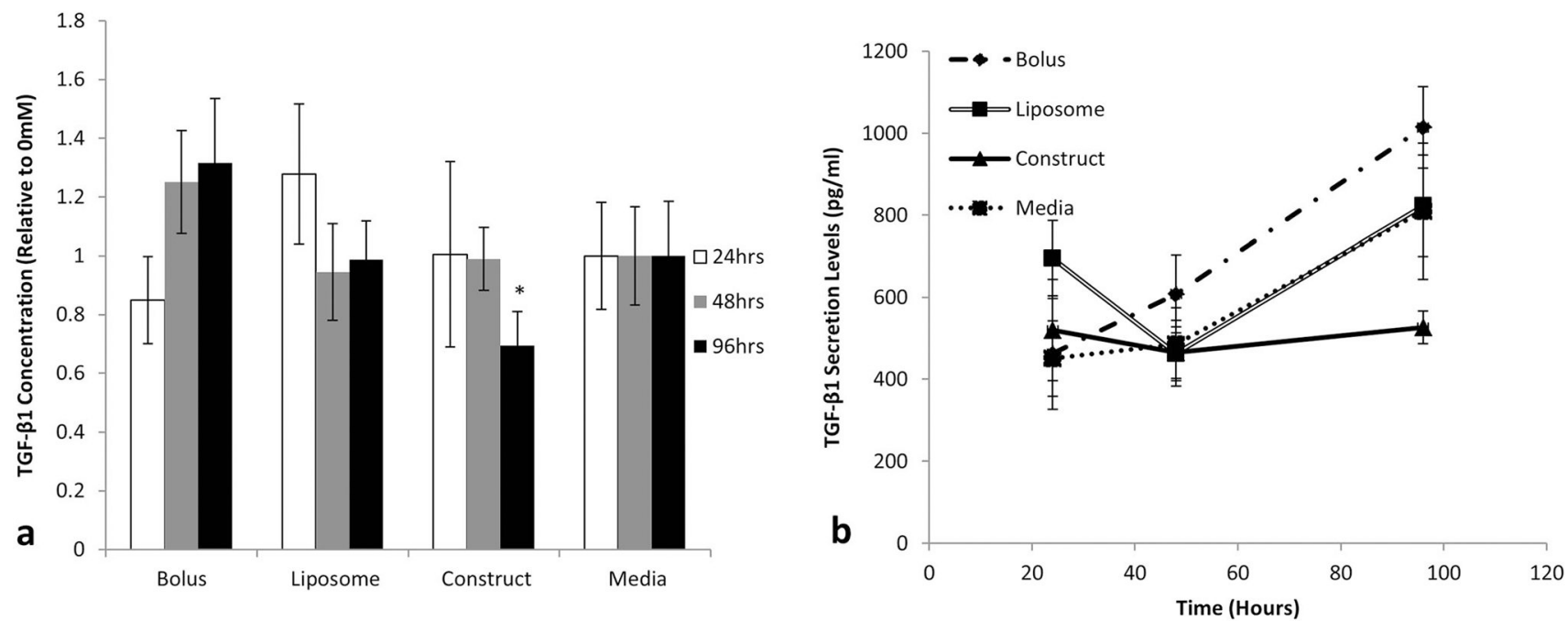

Fig. 6 .

Alginate-liposome construct decreases activated MSC TGF- $\beta 1$ secretion. Levels of TGF- $\beta 1$ quantified in cell culture supernatants collected from MSCs treated for 24, 48, and $96 \mathrm{~h}$ with $1 \mathrm{mM}$ bolus dose of bupivacaine, liposomal bupivacaine, or alginate-liposome construct. a TGF- $\beta 1$ concentrations were normalized to corresponding basal medium controls $(0 \mathrm{mM})$. b TGF- $\beta 1$ secretion over time. Each bar (a) or point (b) is the mean \pm SEM of $n=5-9$ independent observations. Some observations were not detected. An asterisk indicates $<0.01$ bolus at time point 


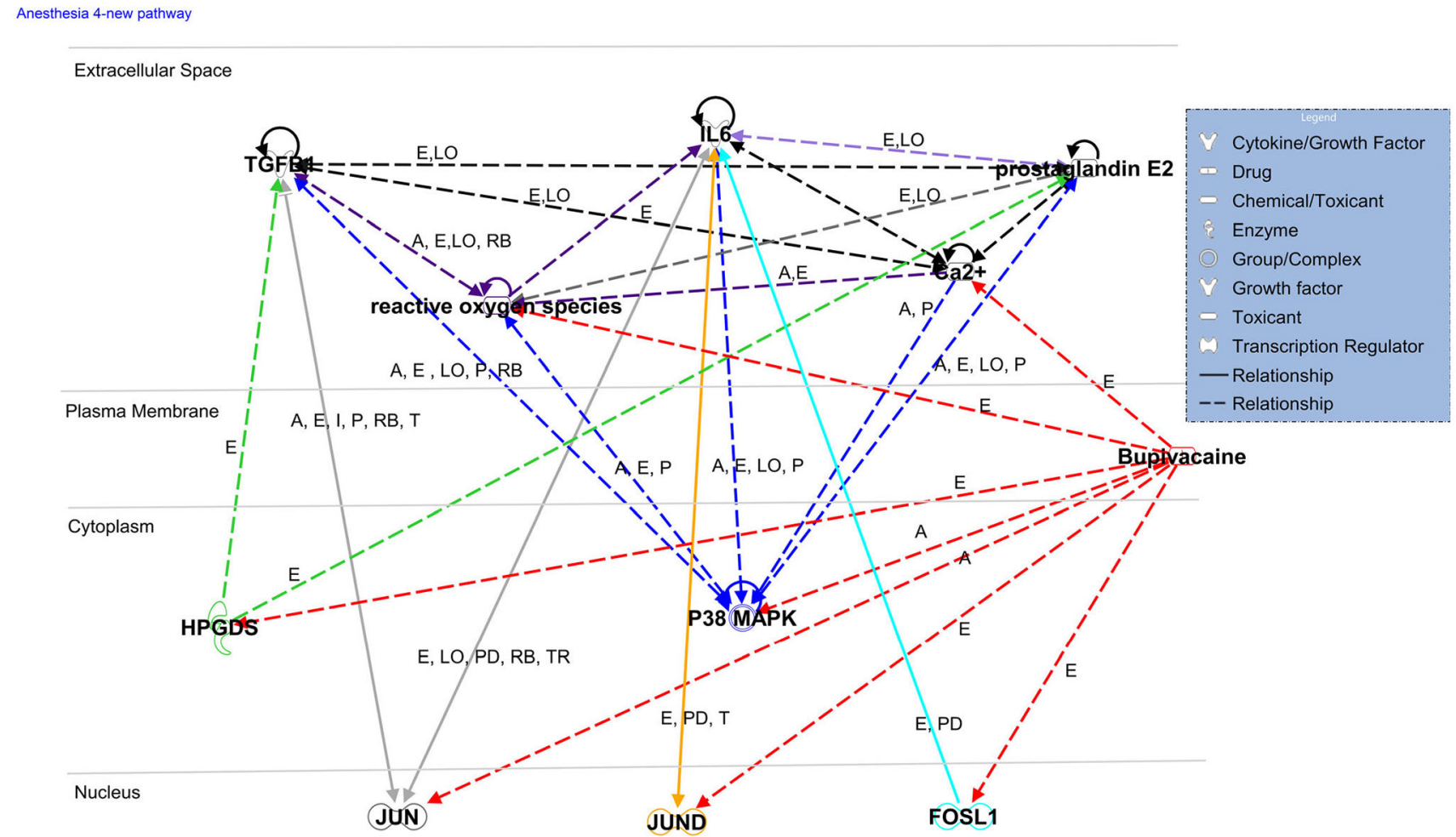

Fig. 7.

Custom pathway established for the predicted molecular signatures of MSC and bupivacaine action. p38 mitogen-activated protein kinases (p38 MAPK) (blue), reactive oxygen species (ROS) (purple), calcium $\left(\mathrm{Ca}^{2+}\right)$ (black), jun-proto-oncogene/activator protein 1 (JUN) (gray), hydroxyprostaglandin dehydrogenase 15-(NAD) (HPGD) (green), junD protooncogene (JUND) (yellow), FOS like 1/AP-1 transcription factor subunit (FOSL-1) (cyan), and PGE2 (lavender) were identified by IPA Pathway Explorer as molecular mediators of bupivacaine action (red) on IL-6, PGE2, and TGF- $\beta 1$. Solid lines indicate a direct relationship between nodes (gene products/molecules) and dashed lines indicate indirect relationships. Letters beside each line indicate the type of interaction between the nodes: A activation, E expression (includes metabolism/synthesis for chemicals), I inhibition, LO localization, $\mathrm{P}$ phosphorylation/dephosphorylation, PP protein-protein interactions, and $\mathrm{T}$ transcription. Relationship legend can be found at http://ingenuity.force.com/ipa/ IPATutorials\# 


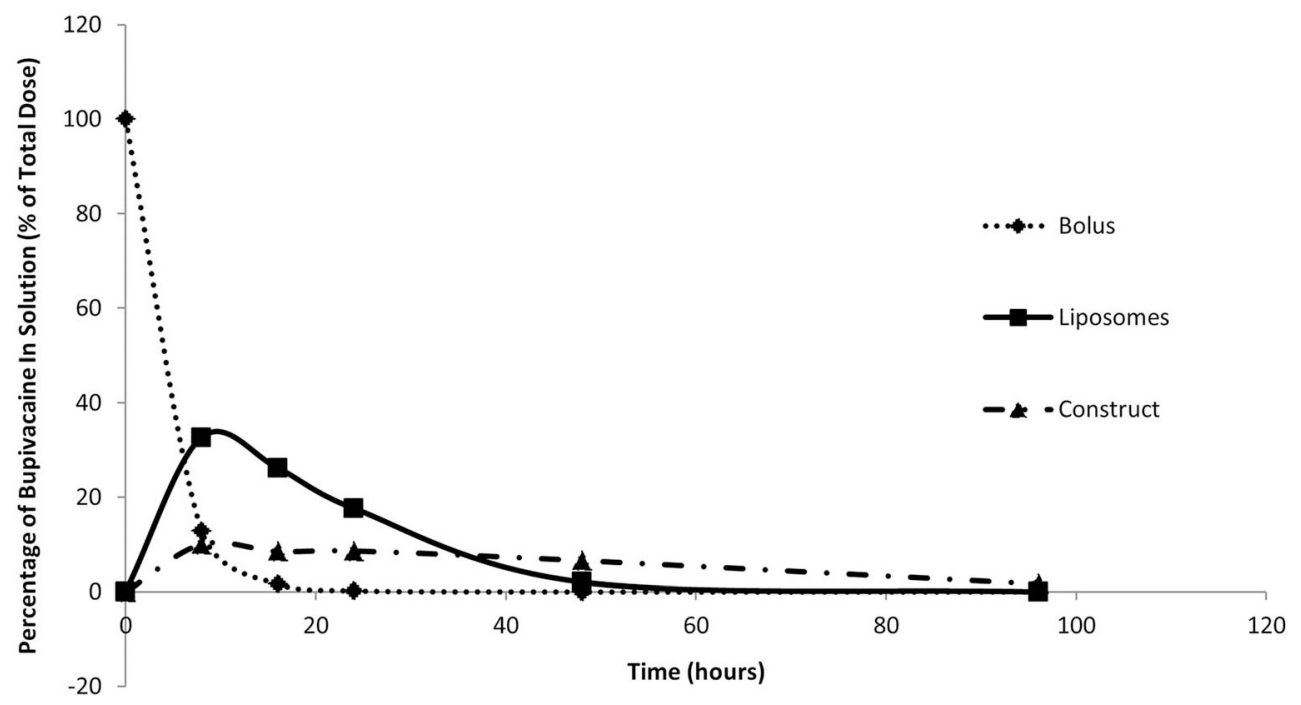

Fig. 8 .

Bupivacaine concentration over time. Using LA concentrations from Maguire et al. [24] and

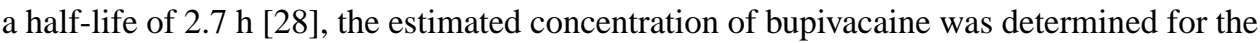
three anesthetic conditions. The percentage of total drug concentration $(1 \mathrm{mM})$ is shown. Concentrations for liposome and construct were determined for 8-h increments until 48 and $96 \mathrm{~h}$, respectively. It was assumed that no additional bupivacaine was released from the bolus and liposome after 0 and $48 \mathrm{~h}$, respectively, based on the data from Maguire et al. [24]. At the different time points, degradation of bupivacaine was determined and added to the value from the previous 8-h segment, which yielded a total drug concentration at the different time points. Concentrations were plotted at $0,8,16,24,48$, and $96 \mathrm{~h}$ 

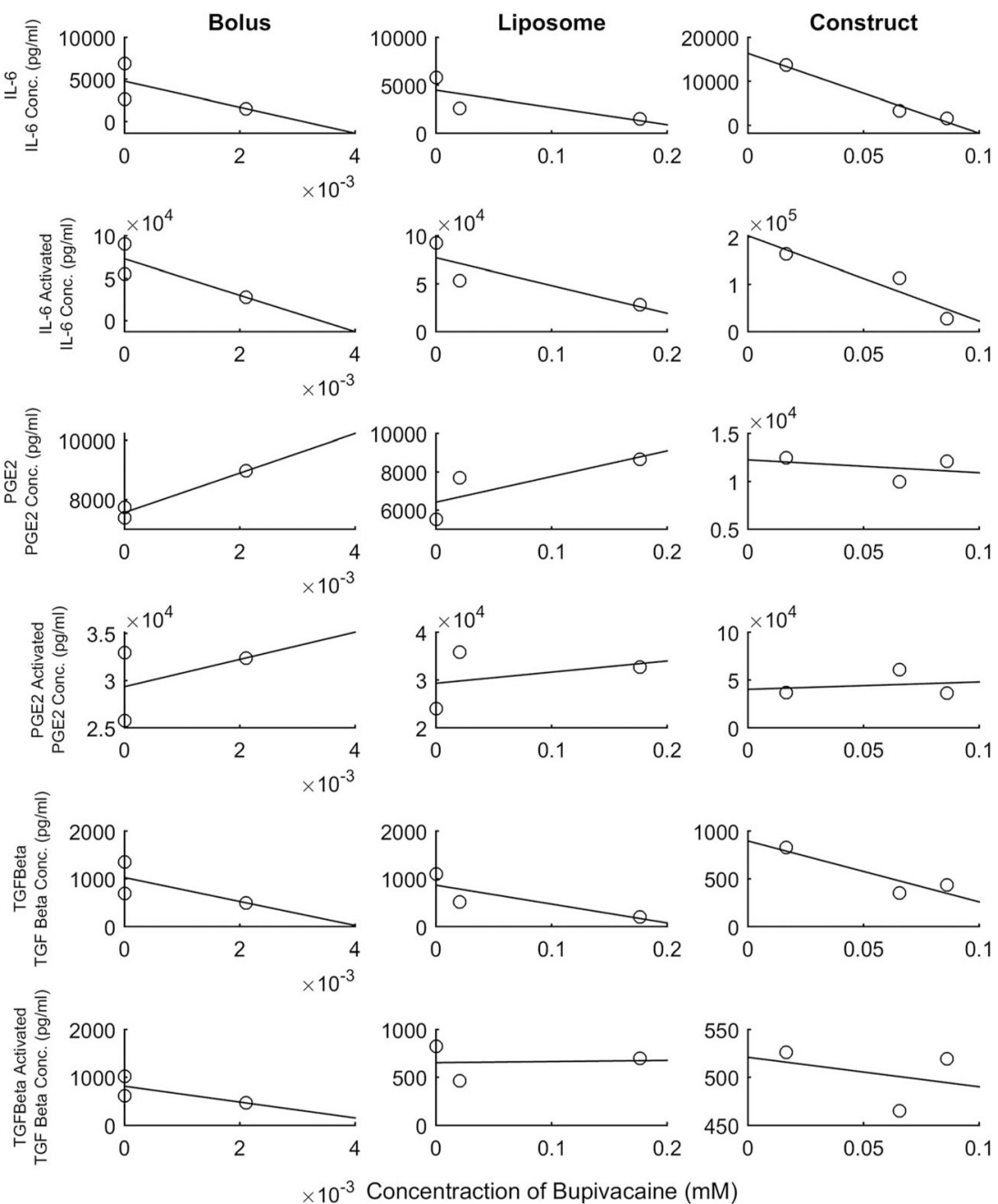

Fig. 9.

Correlations between bupivacaine concentration and analyte concentrations measured by means of ELISA. Linear regression and Pearson's coefficient $(r)$ were derived from standardized data

Drug Deliv Transl Res. Author manuscript; available in PMC 2018 November 06. 

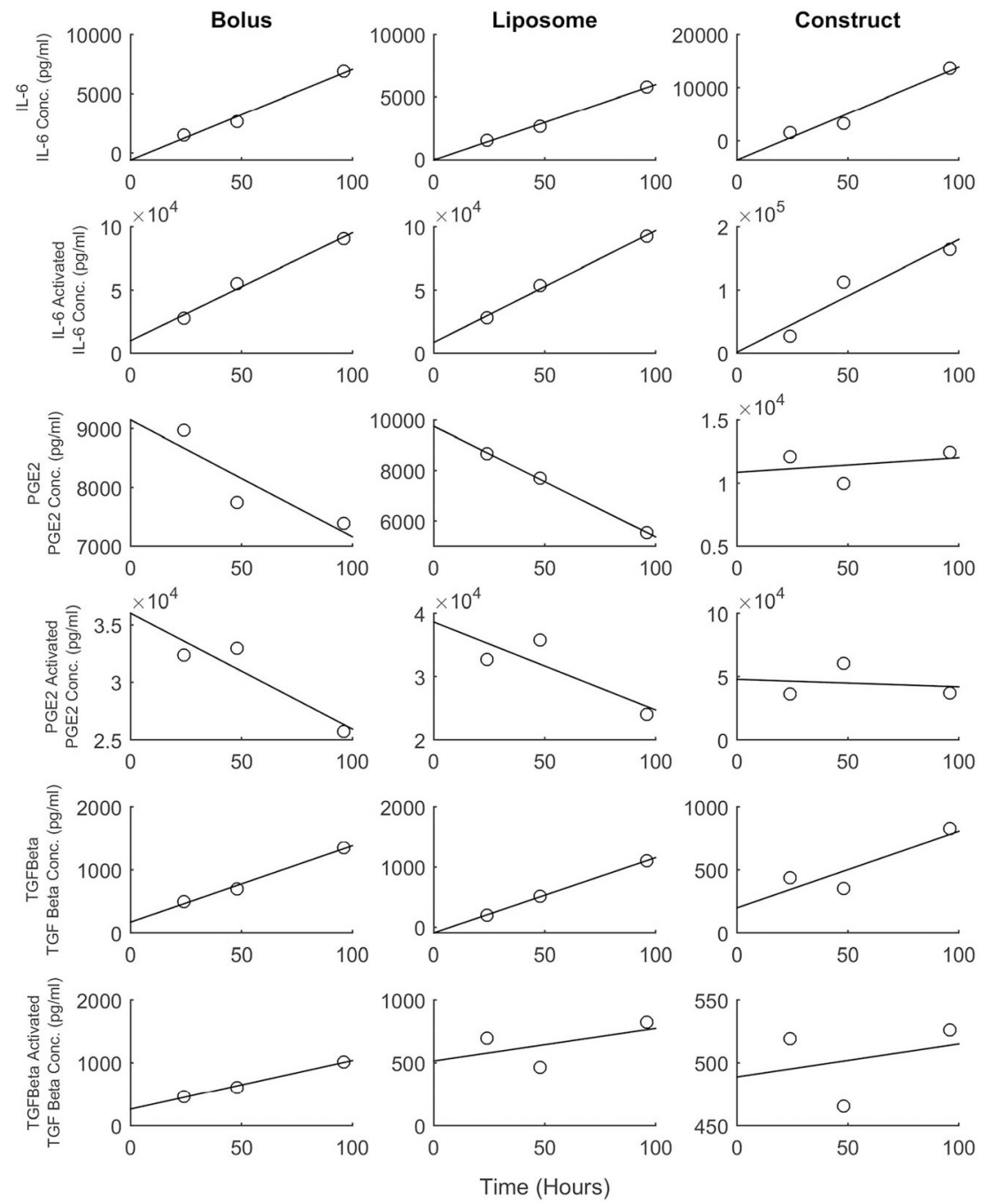

Fig. 10.

Correlations between time and secretion concentrations measured by means of ELISA. Linear regression and Pearson's coefficient $(r)$ were derived from standardized data

Drug Deliv Transl Res. Author manuscript; available in PMC 2018 November 06. 


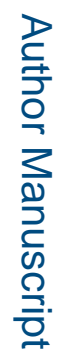

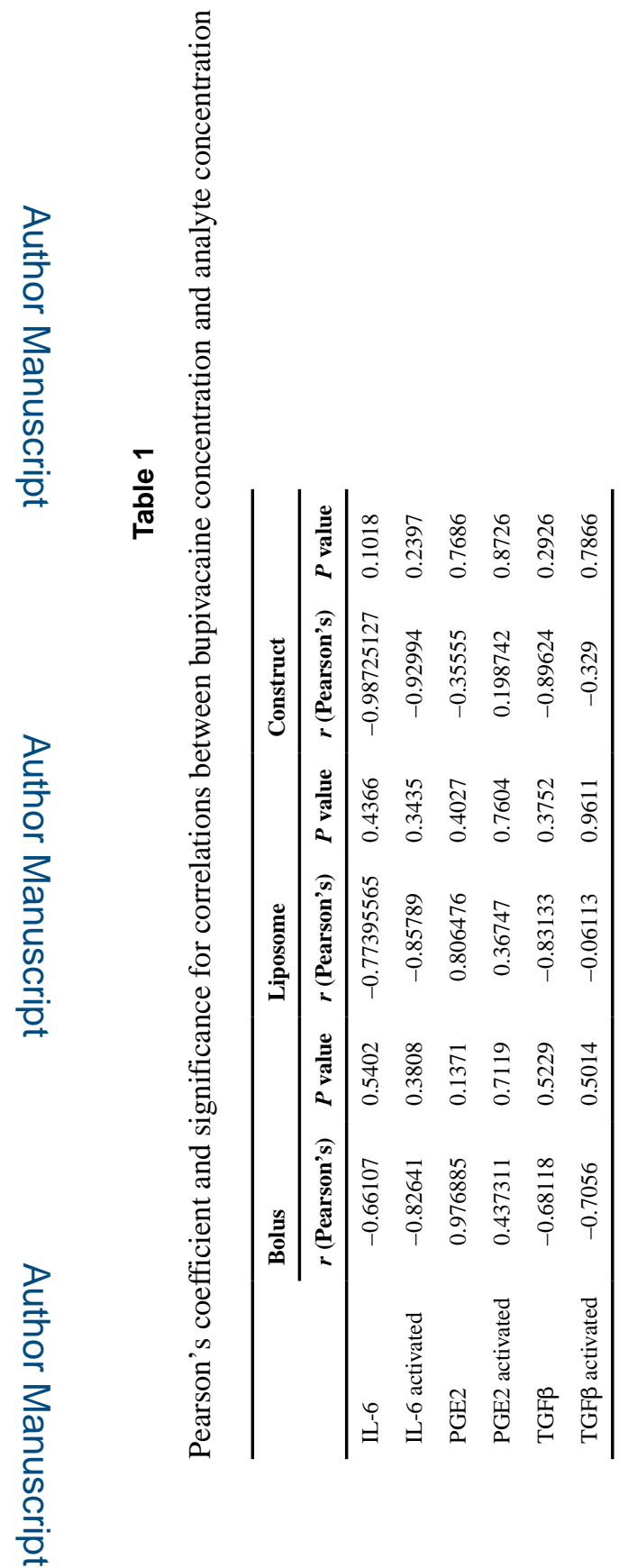

Drug Deliv Transl Res. Author manuscript; available in PMC 2018 November 06. 


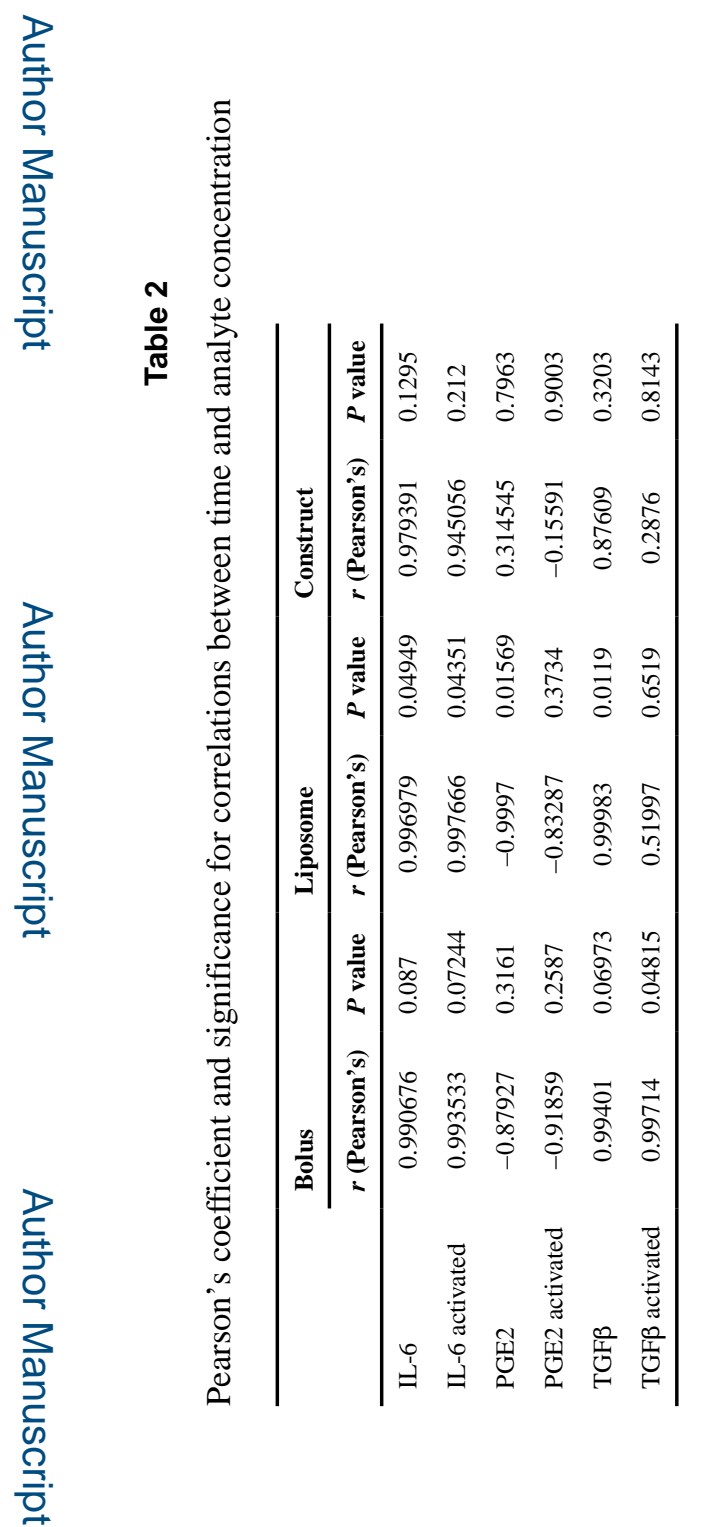

Drug Deliv Transl Res. Author manuscript; available in PMC 2018 November 06. 